\title{
Verifying Existence and Composition of Workflow Activity Patterns in Real Process Models
}

\author{
Carolina Chiao ${ }^{1}$, Lucinéia Heloisa Thom ${ }^{2}$, Cirano Iochpe ${ }^{1}$, Manfred Reichert ${ }^{3}$ \\ ${ }^{1}$ Institute of Informatics - Federal University of Rio Grande do Sul \\ Av. Bento Gonçalves, 9500, 91501-970 - Porto Alegre, RS, Brazil \\ ${ }^{2}$ Institute of Database and Information Systems - University of Ulm \\ Oberer Eselsberg, Geb. O27, 89069 - Ulm, Germany \\ ${ }^{3}$ Faculty of Electrical Engineering Mathematics and Computer Science - \\ University of Twente - 7500 AE Enschede, The Netherlands \\ \{cchiao, ciochpe\}@inf.ufrgs.br, lucineia.thom@uni-ulm.de, \\ m.u.reichertecs.utwente.nl
}

\begin{abstract}
In this paper we present a collection of high level workflow activity patterns based on the semantic of specific business functions (e.g., notification, task execution request, approval). In particular we discuss three pattern samples (approval, unidirectional and decision patterns). Moreover we gather the results of an analysis of their adoption on a wide set of real process models. The analyses showed that the patterns are not only enough but also necessary to model all the 190 process models which were subject of the investigation. We also show and discuss specific sequences or combination of activity patterns which were more often in the process models analyzed. In larger research we apply these patterns as well as the analyses results in the development of a suite for process modeling and normalization.
\end{abstract}

\section{Introduction}

During the last years, companies have been exploring numerous techniques for business process management (BPM) in order to align their information systems in a process-oriented way and to stay competitive in their market. Accompanying this trend the significance of BPM has increased and new quality standards have emerged. According to the quality standard ISO 9001:2000, for example, an organization should be mainly represented by its core business processes rather than by its organizational chart. If BPM is associated with Information Technology (IT), it will become possible to offer additional benefits to the organization, such as: (a) precise and unambiguous description of the existing business processes; (b) improvements regarding the definition of new processes; (c) effectiveness regarding the work coordination between different agents; (d) real time gathering of precise information about process executions; and (e) standardization of business processes.

For (computerized) business processes there exists a variety of fragments which can be understood as self-contained activity blocks with a well-defined semantics [Thom 2006a], [Thom 2006b]. In particular, a certain process fragment (or recurrent business function) may occur several times within one (or different) process 
definition(s). As an example, consider the evaluation process for price adjustment as depicted in Figure 1. This process includes activities with the following partial order: (a) a decision activity (to fix whether the input is a shopping order or not) (b) activity 'send e-mail to manager informing about price adjustment'; (c) activity 'evaluate request of price adjustment'; (d) activity 'notify managers about conclusion of evaluation; (e) activity 'notify managers about automatic approval'; and (f) activity 'prepare request to be sent'. Altogether this process comprises fragments having generic semantics that can be described as patterns such as decision (activity $a$ ), notification (activities $b, d$ and $e$ ), and task execution request (activities $c$ and $f$ ).

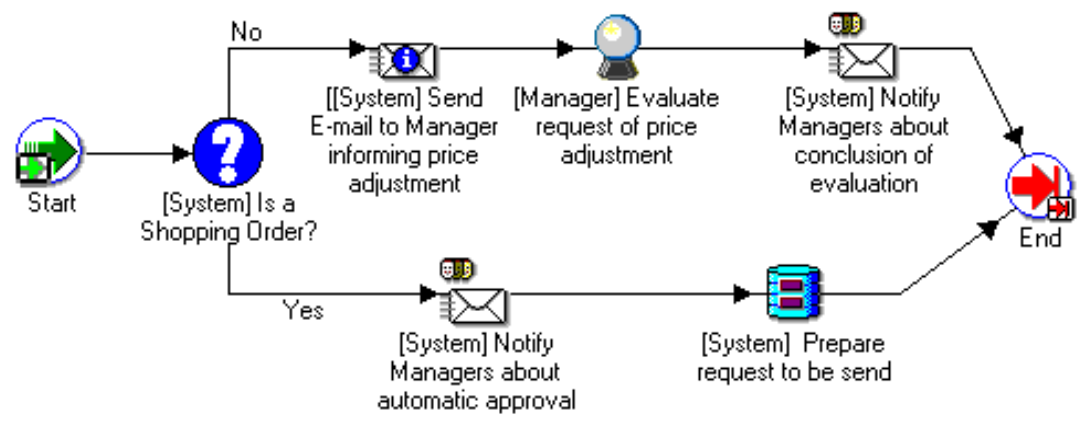

Figure 1: Evaluation process for price adjustment

Recently, research on workflow patterns has emerged in order to increase the reuse of recurring workflow structures. More precisely, different workflow patterns have been proposed for control flow modeling [Aalst 2003], resource management [Russell 2004], data modeling [Russell 2005], service interaction [Barros 2005], workflow exception handling [Russell 2006]. All these pattern sets have in common that they are relevant for implementing a workflow modeling tool, or for defining or evaluating a particular process description language. However, these structural patterns provide only a partial answer to the question what business functions a modeler has to consider repeatedly in various process models.

Usually, such process fragments [Flores 1998], [Medina-Mora 1992], [Malone 2004], [Muehlen 2002] are re-designed for each workflow application. Of course, this lack of reusing model fragments and process knowledge has resulted in high costs and error rates regarding the modeling and maintenance of process-oriented applications. While there is some research reported on how metadata can be organized to manage large-scale modeling projects (cf. [Thomas and Scheer 2006]), to our best knowledge there exists no (empirical) work evidencing the existence of recurrent patterns in real workflow applications. Furthermore, there is no work on which patterns are needed and how good they may support the modeling of at least specific kinds of business processes. Beyond that, contemporary workflow modeling tools do not provide functionalities that enable users to define, query, and reuse such patterns in a proper way.

Concerning this problematic in an earlier work we presented a first approach towards the implementation of workflow activity patterns based on an Event-driven Process Chains (EPCs) tool [Thom 2007a], [Keller 1992]. Recently we proposed a suite for both process modeling and normalization based on the reuse of semantic process patterns (see [Thom 2007b]). In this paper we gather samples of workflow activity 
patterns. However, the main contribution of this paper is the description of a case study where we analyzed 190 real process models from different organizations as well as application domains. We had analyzed not only the frequency of each pattern frequency, within the set of process models, but the frequency of specific sequences of them. Taking the results of the case study we show that the workflow activity patterns do not only exist in real workflow applications, but are also necessary and sufficient to model all the workflow processes which were subject of our investigation. We believe that the use of these patterns together with other existent patterns (e.g., control flow patterns [Aalst 2003]) might not only reduce design effort (e.g., it is a small set of patterns that seems to require little effort to learn) but also optimize and improve the quality of it (e.g., the user can reuse design solutions stored in a knowledge database). This database is part of a larger research where we propose a suite for workflow design based on patterns reuse (cf. [Thom 2007a], [Thom 2007b]).

The outline of this paper is organized as follows: Section 2 gives an overview of the workflow activity patterns. In particular, we discuss the approval, notification and unidirectional as three examples. Section 3 gathers the results of an extensive case study where we investigated the existence of the patterns in 190 process models. In this section we also show how complete is the set of patterns for the design of the 190 processes. In Section 4 we present some patterns combinations by dividing the set of process models in System-Intensive and Human-Intensive processes. This classification is useful to obtain more precise results concerning the kind of processes the patterns are more suitable to be found. Moreover, this information will be used in the development of a knowledge database of patterns. Finally, Section 5 concludes the paper and gives an outlook on future research.

\section{Workflow Activity Patterns}

A Workflow ACtvity Pattern refers to the description of a recurrent business function frequently found in business processes (e.g., notification, decision, approval). We derived a set of 7 patterns from an extensive study based on the literature (cf. [Thom 2006a] and [Thom 2006b]). The patterns are approval, question-answering, unidirectional and bi-directional performative, information, notification and decision patterns.

A block activity is suitable to represent each pattern according to [WfMC 2005]. The block activity concept is particularly suited because it allows to encapsulate the well-defined semantics and to represent their atomic characteristics. This means that all activities defined inside a block activity pattern must be completed before the superordinated workflow can continue its execution.

We describe three example patterns with the Business Process Modeling Notation (BPMN). The complete set of patterns can be found in [Thom 2006a]. For each pattern we describe "context", "problem", "forces" and "Solution". The solution includes one "design choice". Currently we are working on the improvement of the patterns documentation. We are giving examples of the patterns use, defining desigh choices and describing how they can be implemented. 
Pattern1: APPROVAL

Context: A document must be evaluated by one or more organizational role.

Problem: How to model a human decision-making in the workflow process?

\section{Forces:}

- In case of multi-approvals (concurrent), the number of times that the decision-making activity is repeated may vary depending on the level of centralization of authority (less or more) as well as the direct supervision of work existent in the organizational unit(s) where the process is executed.

- The decision-making activity is generally performed by a human.

- The decision-making activity must have more than one kind of response (e.g. approval and reproval).

Solution: To include in the workflow, a human activity that characterizes a point of decision-making on the sub-product in question (e.g. a document requiring approval). This decision-making activity will be repeated according to the level of centralization existent in the organizational units where it is executed. Figure 3 shows a single approval.

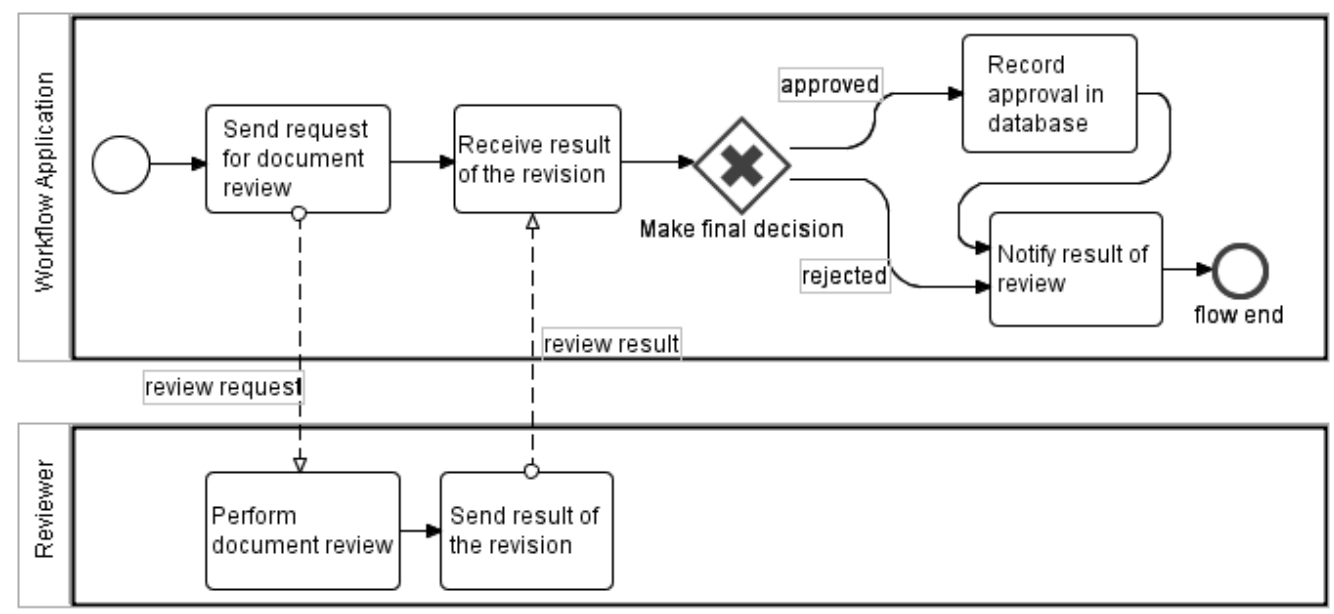

Figure 3: Approval pattern

In Fig. 3 an organizational role reviewer performs a document review either resulting in an approval or disapproval. In case of multi-approvals, it would be necessary concurrent activities. The "Make final decision"(cf. Figure 3) would be executed only when all reviewers had performed their revisions The revisions would be then performed multiple times in parallel (concurrent) or in sequence (iterative) according to the number of organizational roles specified or until disapproval occurs. Generally, the number of organizational roles is connected to the level of centralization (in high positions of the organization) with respect to decision-making.

\section{Pattern 2: Unidirectional Performative}

Context: In a workflow, there is a moment that the process must request the execution of an activity to the system or to an organizational role involved in the process. 
Problem: While modeling the workflow, how to send a request without waiting for the result of the activity execution?

\section{Forces:}

- The receiver's response is not required.

- The process must keep its execution without waiting for the activity to be completed.

- The request can be done to the system or a human.

Solution: To include in the workflow model the sequence of activities (see Fig. 4) representing the unidirectional performative message. The sequence of activities comprises the generation of a work item in the receiver worklist. However, the workflow does not wait for a receiver response to continue execution.

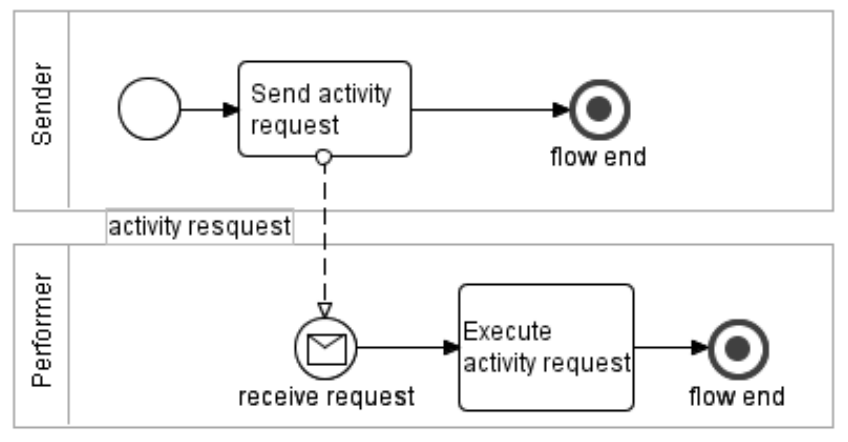

Figure 4: Unidirectional Performative Pattern

A sender uses unidirectional performative messages to request the execution of an activity from a receiver. As shown in Fig. 4, an activity execution request results in a work item being assigned to a receiver (i.e., a specific workflow participant responsible for the activity execution). After that, the process may continue execution without waiting for a response.

\section{Pattern 3. NOTIFICATION}

Context: During the process execution, some specific events have more relevancy such that the process must inform some organizational roles about them.

Problem: While modeling the workflow, how can we keep the involved roles informed about some process instance events?

\section{Forces:}

-The notification must be sent by an electronic way.

-The process does not have to wait for a reading response to continue execution.

-The notification generally contains the status of a process activity (e.g., completed, document approved, rejected).

Solution: To include in the workflow the structure concerning the notifying and do not wait for response. Figure 5 shows how this structure works. There's the sending of the notification and its receiving. These activities must inform the involved roles in the 
process about news inherent to the respective workflow, such as, for example, the approval (or reproval) of a document, or a task that has achieved its timeout.

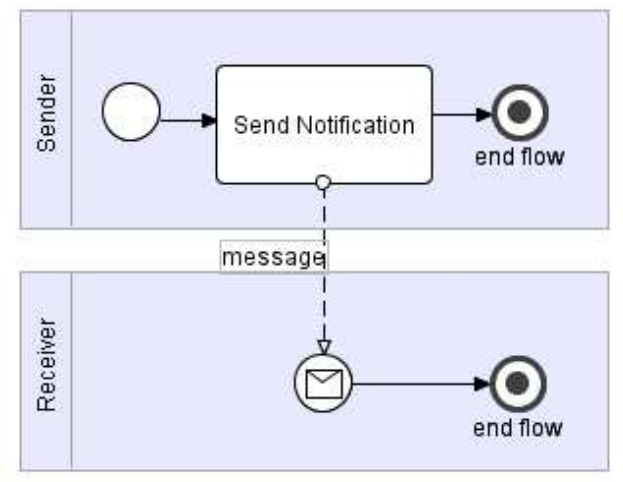

Figure 5: Notification Pattern

\section{Evidencing the Existence of Workflow Activity Patterns by Analyzing Real-World Process Models}

With the goal to search for the existence of the workflow activity patterns in real-world applications we analyzed 190 process models. These workflows have been modeled with the Oracle Builder tool and have stemmed from 12 different organizations related to different application domains. Notice that we analyze process models and not instances or logs generated by the execution of them. We do that because the semantic of the activities is important to the identification of the processes. Table 1 characterizes the process models subject of our analyses.

\begin{tabular}{|l|l|l|l|}
\hline $\begin{array}{c}\text { Size of the } \\
\text { company }\end{array}$ & \multicolumn{1}{|c|}{ Kind of decision-making } & \multicolumn{1}{|c|}{$\begin{array}{c}\text { Examples of process models we } \\
\text { analyzed }\end{array}$} & $\begin{array}{c}\text { Number of process } \\
\text { models analyzed }\end{array}$ \\
\hline 1 small & Decentralized & Management of internal activities & 17 \\
\hline 1 large & Decentralized & TQM and management of activities & 11 \\
\hline 6 large & Centralized & $\begin{array}{l}\text { TQM; control of software access; } \\
\text { document management }\end{array}$ & 133 \\
\hline 4 large & $\begin{array}{l}\text { We had no access to } \\
\text { information about these } \\
\text { companies }\end{array}$ & $\begin{array}{l}\text { Help Desk, User feedback; } \\
\text { document approval }\end{array}$ & 29 \\
\hline
\end{tabular}

Table 1: Core characteristics of the analyzed process models

We have obtained the following results from the case study, i.e. the process models analyses:

a) evidence with high probability that the workflow activity patterns exemplified in this paper exist in real workflow applications;

b) evidence that the set of patterns is both necessary and sufficient to model all 190 process models analyzed; and

c) identification of sequence of patterns based on the classification of the process models into Human-Intensive and System-Intensive [Le Clair 2007]. 


\subsection{Method Used to Analyze the Workflow Processes}

For each activity pattern we calculated its support value $(S)$. In the context of this paper, $S$ represents the number of occurrences of each pattern $(P)$ in a set of 190 process models. For those models comprising more than one occurrence of the same pattern just one was considered. The following formula was considered to calculate the support:

\section{Where:}

$\mathrm{S}=\frac{\mathrm{F}(\mathrm{P})}{\mathrm{T}_{\mathrm{T}}}$

$\mathrm{F}(\mathrm{P})=$ frequency of a specific workflow activity pattern in the total set of process models

$\mathrm{T}_{\mathrm{T}}=$ total number of process models

Initially, we identified and annotated workflow activity patterns in all process models we analyzed. Afterwards, for all process models we counted the number of occurrences of each pattern. The obtained result then was divided by the total number of analyzed process models (i.e. 190 in our case). Accordingly, the $(P)$ for this calculation corresponds to a specific pattern while $T_{T}$ means the set of process models.

\subsection{Frequency of Workflow Activity Patterns in Real-World Process Models}

The UNIDIRECTIONAL and BI-DIRECTIONAL PERFORMATIVE PATTERNS, DECISION PATTERN, NOTIFICATION PATTERN and INFORMATIVE PATTERN are not dependent on specific application domains or organizational structure aspects. This fact mainly explains why they were identified with high-probability in practically all process models analyzed. The same applies to the APPROVAL PATTERN. This can be explained by the high degree of centralization on decisionmaking existing in the organizational units for which we analyzed their processes. This high centralization implies the use of approval activities. Besides that, several process models belong to applications related to approval contexts. By contrast, most of the analyzed process models do not comprise QUESTION-ANSWERING activities. The use of question-answering activities depends on the kind of process model being modeled. It is used in those processes where some question can occur. Figure 6 graphically illustrates the frequency of each activity pattern in the set of process models analyzed.

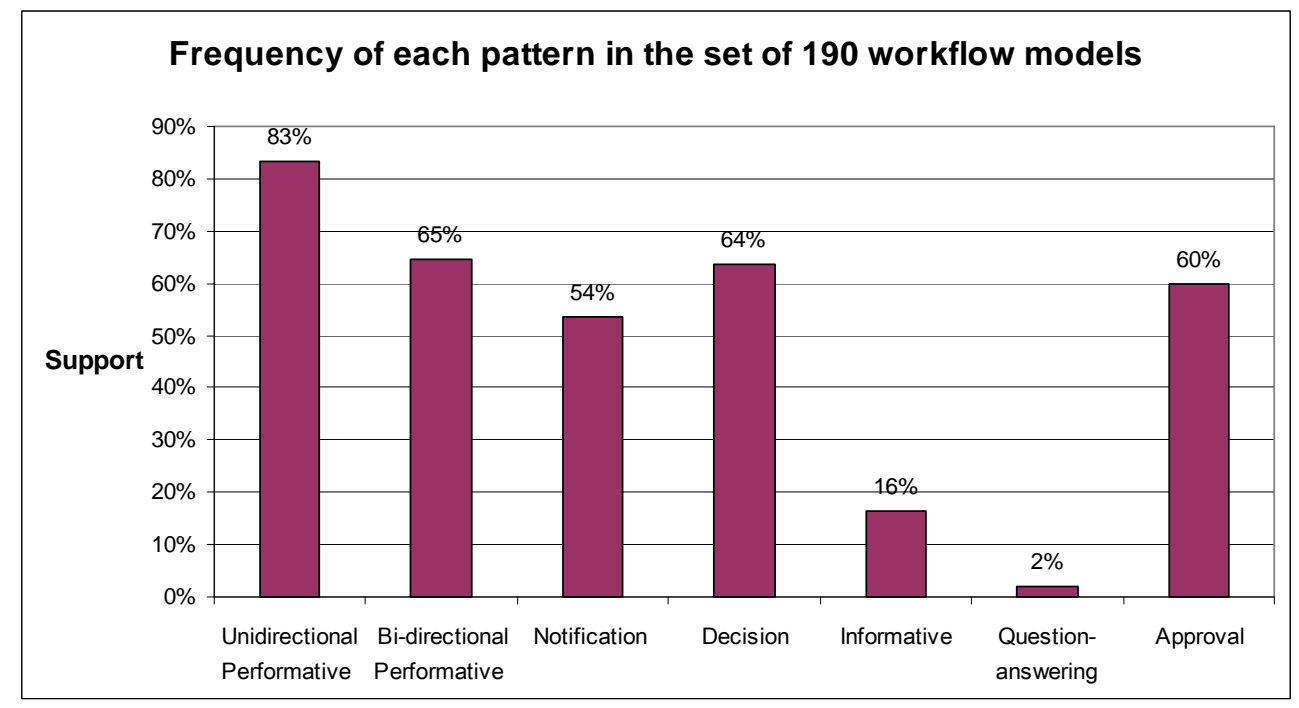

Figure 6: Frequency of the workflow patterns in real-world process models 


\subsection{Discussing the Completeness of the Workflow Activity Patterns for Workflow Modeling}

The main goal of the study case presented in this paper is the measurement of the frequency which each one of the workflow activity patterns happens in the set of process models that has been analyzed. This was done in order to verify whether these business functions (e.g., task execution request, notification) could really be considered as activity patterns with high probability of reuse in business as well as workflow process design at least those with similar characteristics of the processes we analyzed.

While some activity patterns are identified only by the analysis of the activity description (e.g., decision, approval and notification patterns), others required a more detailed analysis. For instance, the INFORMATIVE PATTERN is identified in activities where the user provides an information to the system (e.g., by the fulfillment of a field in the context of an activity) (cf.[Thom 2006a]). In the case of the UNIDIRECTIONAL and BIDIRECTIONAL PERFORMATIVE PATTERNS, both the activity description and its execution result (i.e., mandatory or not to trigger the next activity in the process) are important to measure how often the patterns occur.

What really surprised us is the fact that all analyzed workflow processes can be defined as a composition of the investigated patterns (cf. Figure 7 for an example). That is, the set of workflow activity patterns is necessary and sufficient to design all 190 real process models that were subject of our analyses. In each process, a specific activity pattern may appear zero or more times combined with other patterns.

This fact can be considered as a very important one which points out to new questions to be investigated as part of a future work. For instance, how much could this set of patterns be helpful if it was to be integrated into a workflow design tool? One could think of an intelligent software module which relies on both a workflow activity patterns repository in order to help designers to complete their workflow design. First initiative in this approach we present in [Thom 2007a] and [Thom 2007b]. Figure 7 shows a process model sample where all activities match to some activity pattern.

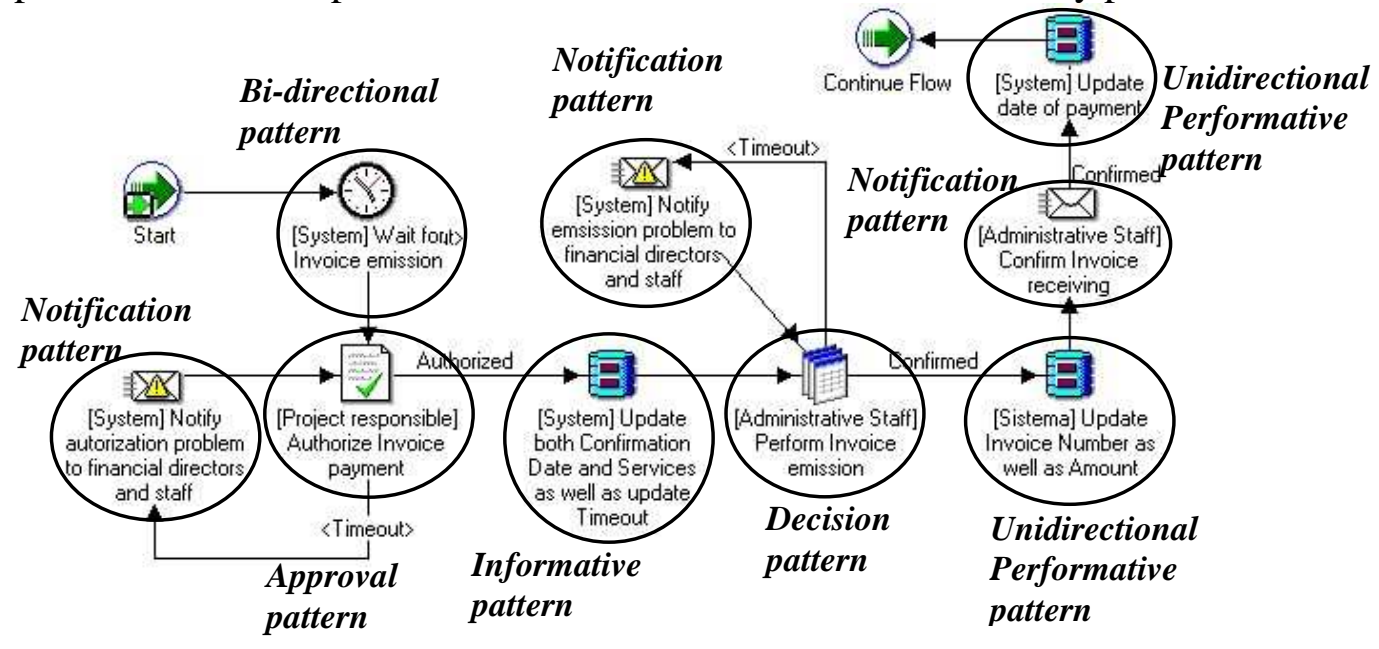

Figure 7: A payment process built up exclusively from the combination of workflow activity patterns 


\section{Identifying Sequences of Workflow Activity Patterns in Real Process Models}

Some process fragments can occur many times in the same process definition [Thom 2006a]. Each time a specific fragment occurs, there may have successive process fragments that can also occur with more frequency than other fragments.

With the objective of evidencing which are the workflow activity patterns that succeed with more frequency one specific pattern, we analyzed 151 of the 190 process models, which were subject of our case study. Again we analyzed the process models and not the execution logs.

Initially we made a preliminary investigation where we analyze, for each process activity and its respective workflow activity pattern associated, what was the subsequent(s) pattern(s) that follow them. By doing that, we noticed that most of the patterns pairs had low support value and confidence. Figure 8 illustrates one of the obtained results of this first analysis. Notice that even the most frequent pair of this example (DECISION PATTERN $\rightarrow$ NOTIFICATION PATTERN) has a low probability (29\%).

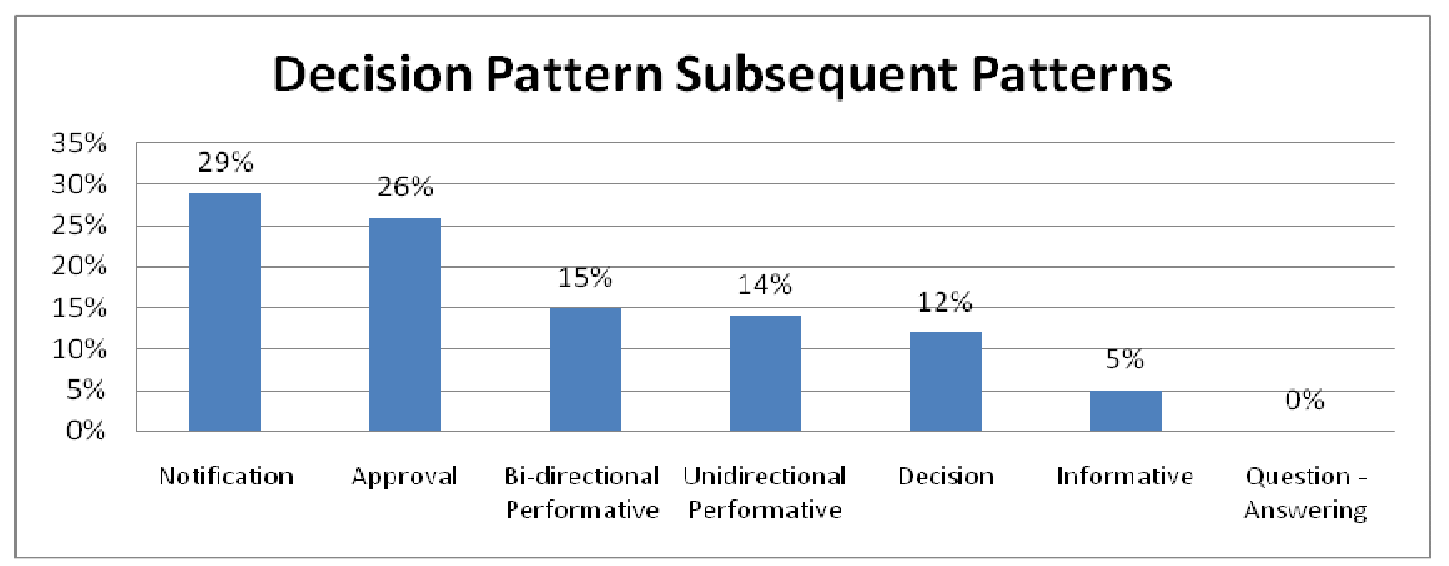

Figure 8: Decision Pattern Subsequent Patterns on the preliminary analyses

By analyzing the process models, we observed that some kinds of activity patterns, and their respective pairs, appear with more frequency in determined types of processes. We noticed that process including decision-making activities (i.e., approvals) present higher probability of being followed by the NOTIFICATION PATTERN. This fact is mainly explained because the organizational roles involved in the processes must be notified about the result of the approval process.

In order to increase the support and confidence of the pattern pairs, we decided to classify the processes into business process categories. For that, we studied some of the main classifications found in the literature [Hammer 2001], [Harrington 1991], [Dowson 1987], [Leymann 1999]. However, most of them classify processes by application domains. Accordingly, those approaches were not feasible to our analyses because the set of processes we were investigating do not cover all the categories described on these works. We chose then the classification proposed by Le Chair where processes are divided into system-intensive and human-intensive [Le Clair 2007].

The system-intensive processes are characterized by being handled on straightthrough basis, which means that there is minimal or no human touch and few 
exceptions. The human-intensive processes require people to get work done by relying on and interacting extensively with business applications, databases, documents and other people. This type of process requires human intuition or judgment for decisionmaking during individual steps.

Having this clear separation between the processes, we divided the set of studied process models into processes that have human interaction and processes that don't have human interaction. The result of this classification is 31 processes system-intensive and 120 processes human-intensive. Figure 9 shows the results of this investigation over decision patterns in system-intensive processes. Notice that the probability of having a DECISION PATTERN $\rightarrow$ NOTIFICATION PATTERN pair has increased to $50 \%$.

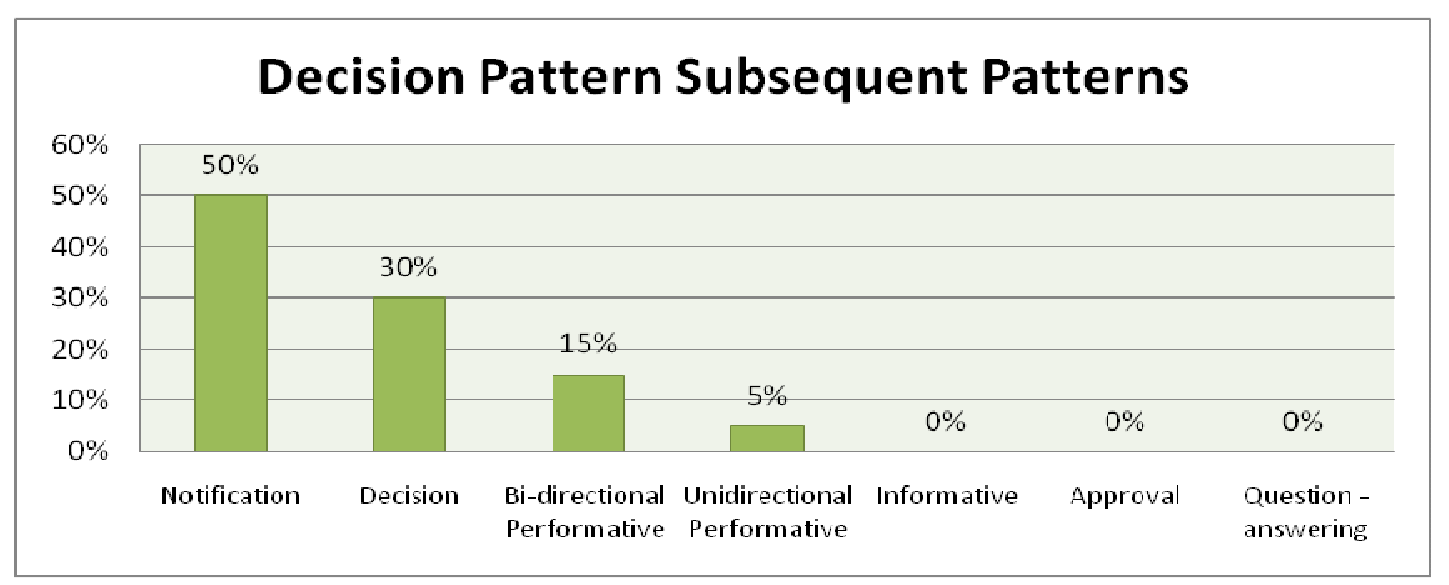

Figure 9: Decision pattern subsequent patterns by analyzing only the systemintensive processes

\section{Conclusions}

In this paper we presented examples of workflow activity patterns which can be used to design business process and workflow models respectively. In particular we reported the results of empirical studies we had performed in order to measure the frequency with which each workflow activity pattern occurs within a set of 190 process models. This analysis was accomplished in order to verify whether specific business functions frequently found in business processes (e.g., task execution request, notification, approval) may be considered as patterns with high probability for reuse. We also showed that by dividing the processes we analyzed into system-intensive and humanintensive it was possible to identify pairs of patterns which were more frequently present in the processes.

The main advantages of this approach can be summarized as follows: (a) the completeness and necessity of the workflow activity patterns has been evidenced at least for the design of the process models subject of our analyses; (b) the patterns are toolindependent, which make them easier to be adapted for any business process modeling tool; (c) it is a small set of patterns which may reduce complexity in user learning;

The result of the case study we presented in this paper will be used in the development of a suite to the analysis and properties verification in workflow specification (e.g., correctness, completeness, deadlock, processes equivalence, livelock, model checking). This suite will have a knowledge database that will store the workflow 
activity patterns as well as the results of our case study. We consider that this knowledge database will help on matching the patterns with business process extracted from legacy code and will help the user on designing the business process from scratch. By using the pattern pairs, we can help the user by suggesting which pattern is better combined with the one that he/she has already modeled.

As future work we intend to perform additional analyses considering process models from different application domains (e.g., health insurance and automotive). Our goal is to identify not only pairs of co-related patterns but also sequences of workflow activity patterns, including more than three patterns in sequence. In this context we also intend to continue studying the workflow classifications so that we can find more specific classification and with smaller granularity to divide the set of processes. A less generic classification will be useful when we try to converge on the user needs using just a few steps. Finally we consider making an experiment for comparing process modeling with and without workflow activity patterns support.

\section{Acknowledgements}

The authors would like to acknowledge the Coordination for the Improvement of Graduated students (CAPES), the Institute of Databases and Information Systems of the University of Ulm (Ulm, Germany) and the Informatics Institute of Federal University of Rio Grande do Sul (Porto Alegre, Brazil).

\section{References}

Aalst, W.M.P. van der. (2003) "Workflow Patterns". In Distributed and Parallel Databases, 14(3), pages 5-51.

Bernstein, P. A.; Hadzilacos, V.; Goodman, N. (1987) "Concurrently Control and Recovery in Database Systems". Reading: Addison-Wesley. 370p.

Barros, A.; Dumas, M.; Hofstede, ter A. (2005) Service Interaction Patterns. In W.M.P. van der Aalst, editor, Proceedings of the 3rd International Conference on Business Process Management, v. 3649 of Lecture Notes in Computer Science, pp. 302-318. Springer-Verlag.

Dowson, M. (1987) Interation in the Software Process Review of the 3rd International Software Process Workshop. In Proceedings of the 9th international Conference on Software Engineering (Monterey, California, United States). International Conference on Software Engineering. IEEE Computer Society Press, Los Alamitos, CA, 36-41.

Flores, F.; Graves, M.; Hartfield, B.; Winograd, T. (1988) "Computer Systems and the Design of Organizational Interaction". ACM Transactions on Information Systems, 6, 2 (Apr. 1988), 153-172.

Hammer, M.; Champy, J. (2001) "Reengineering the Corporation: A Manifesto for Business Revolution". HarperBusiness. 257p.

Harrington, H. J. (1991) "Business Process Improvement: The Breakthrough Strategy for Total Quality, Productivity, and Competitiveness". McGraw-Hill. 274p. 
Keller, G.; Nüttgens, M.; Scheer; A.-W. (1992) Semantische Prozessmodellierung auf der Grundlage "Ereignisgesteuerter Prozessketten (EPK)". Heft 89, Institut für Wirtschaftsinformatik, Saarbrücken, Germany.

Le Clair, C.; Teubner, C. (2007) The Forrester Wave: Business Process Management For Document Processes, Q3 2007.

Leymann, F.; Roller, D. (1999) "Production Workflow: Concepts and Techniques". Prentice Hall. 479p.

Malone, T. W.; Crownston, K.; Herman, G. A. (2003) "Organizing Business Knowledge: The MIT Process Handbook". MIT Press.

Medina-Mora, R.; Winograd, T.; Flores, R.; Flores, F. (1992) "The action workflow approach to workflow management technology". In Proceedings of the 1992 ACM Conference on Computer-Supported Cooperative Work. 281-288.

Muehlen, M. zur. (2002) "Workflow-based process controlling": foundations, design, and application of workflow-driven process information systems. Berlin: Logos Verlag.'299 p.

Russell, N.; Aalst, W.M.P. van der; Hofstede, A.; Edmond, D. (2004) "Workflow Resource Patterns: Identification, Representation and Tool Support". In Lecture Notes in Computer Science, (2005), 216-232.

Russell, N.; Hofstede, A. H. M Ter; Edmond, D. (2005) "Workflow Data Patterns". In: Proceedings of the 24th ER, pp. 353-368.

Russell, N.; Aalst, W.M.P. Van Der; Hofstede, A. Ter. (2006) "Workflow Exception Patterns". In: Proceedings of 18th CAiSE, 18, p.288-302.

Thom, L. H.; Iochpe, C.; Amaral, V. L. do; Viero, D. M. (2006a) "Toward block activity patterns for reuse in workflow design". In: Workflow Handbook of WfMC, pp. 249-260.

Thom, L. H. (2006b) "A Pattern-based Approach for Business Process Modeling". PPGC: Porto Alegre. Available at: http://www.biblioteca.ufrgs.br/bibliotecadigital/. (Thesis).

Thom, L. H; Lau, J.M.; Iochpe, C.; Mendling, J. (2007a) "Extending Business Process Modeling Tools with Workflow Pattern Reuse". In: Proceedings of 9th International Conference on Enterprise Information Systems, Funchal, Madeira, Portugal.

Thom, L. H., Chiao, C. M., Iochpe, C., Hess, G. N., Nascimento, G. S., Reichert; M (2007b): "Towards an Intelligent Designer based on the Reuse of Workflow Patterns". In: $1^{\text {st }}$ Brazilian Workshop on Business Process Management to be held in conjunction with WEBMEDIA 2007, Gramado, Brazil.

Thomas, O.; Scheer, A.-W. (2006) "Tool Support for the Collaborative Design of Reference Models - A Business Engineering Perspective”. In: 39th HICSS-39, CDROM / Abstracts Proceedings. .

Workflow Management Coalition, 2005. "Process Definition Interface": XML Process Definition Language. Doc. Number: WFMC-TC-1025. (2005). 